\title{
Review: good evidence supports use of polyethylene glycol and tegaserod for constipation
}

Ramkumar D, Rao SS. Efficacy and safety of traditional medical therapies for chronic constipation: systematic review. Am J Gastroenterol 2005;100:936-71.

\section{Q How effective and safe are various medical therapies in patients with chronic constipation?}

\section{METHODS}

Data sources: Medline (1966-2004) and bibliographies of
relevant studies and reviews.
Study selection and assessment: randomised controlled trials
(RCTs) that compared medical therapies with placebo or
compared 2 separate agents in adults with constipation.
Assessment of study quality was done using a 5 point score (5 =
highest quality) based on randomisation procedure, allocation
concealment, blinding, and completeness of follow up.
Outcomes: stool frequency, stool consistency, straining, use of
additional laxatives, ease of defecation, and side effects.

\section{MAIN RESULTS}

Laxatives evaluated were osmotic laxatives (eg, polyethylene glycol [PEG], lactulose, milk of magnesia, and sorbitol), irritant and stimulant laxatives (eg, senna and bisacodyl), bulking agents (eg, psyllium [ispaghula], methylcellulose, bran, celandine, and aloe vera), softening or wetting agents (eg, docusate and poloxalkol), and other agents (eg, misoprostol, cisapride, colchicines, and tegaserod). Levels of evidence (good, fair, and poor) and classifications of recommendations (A [good evidence supports], B [moderate evidence supports], C [poor evidence supports], D [moderate evidence against], and E [good evidence against]) were assigned to each drug or drug class. The table summarises the results of trials with sufficient evidence to make a recommendation. Good evidence supports the use of PEG or tegaserod, and moderate evidence supports the use of lactulose and psyllium. Most side effects did not preclude use of agents.

For correspondence: $\operatorname{Dr} \mathrm{S} S \mathrm{~S}$ Rao, University of lowa Carver College of Medicine, lowa City, IA, USA. satish-rao@uiowa.edu

Source of funding: Novartis Pharmaceuticals.

\section{CONCLUSIONS}

In patients with constipation, good evidence supports the use of polyethylene glycol and tegaserod, and moderate evidence supports the use of lactulose and psyllium. A paucity of placebo controlled trials exists to evaluate medical therapies for constipation.

\section{A modified version of this abstract appears in ACP Journal Club.}

\section{Commentary}

C onstipation is defined by the passage of infrequent hard stool and is associated with bloating, flatulence, abdominal pain, and a feeling of incomplete evacuation. Specific patient groups, such as those with terminal illness, are at high risk of constipation, but the condition is also a common reason for consultation in general practice, especially for the young and the very old. ${ }^{\text {. }}$

Bowel care tends to be a local procedure; it is not unusual to find that different wards or units have their own bowel management protocols. Searching the internet with the terms "bowel care nursing" returns 677000 hits of stepped processes, ranging from diet and fluids, to therapeutic agents, to enemata. The review by Ramkumar and Rao provides a basis to shift bowel care and treatment of constipation from the domain of ritual to evidence-based practice. Although the review was restricted to published English language studies indexed in 2 databases, the overall findings remain remarkably similar to those of a previous review; ${ }^{1}$ namely that generally limited evidence supports a range of laxatives, and the need for good quality trials remains.

The review by Ramkumar and Rao found good evidence supporting the use of polyethylene glycol and tegaserod. When considered with the findings of the previous review, ${ }^{1}$ this provides the evidence for a stepped approach to bowel care, involving bulking agents, drug therapy, and enemata. However, adequate hydration, diet, and exercise should also be encouraged when possible, as these are known to affect constipation. Further trials testing strategies to prevent constipation, particularly in high risk groups, are warranted.

Jane P Joy, RGN, MSc, PGDE Nursing \& Midwifery School, University of Glasgow Glasgow, UK

1 NHS Centre for Reviews and Dissemination. Effectiveness of laxatives in adults. Effective Health Care 2001;7:1-12.

Randomised controlled trials (RCTs) of medical therapies for constipation

\begin{tabular}{llll}
\hline Drug class & Drug & Number of RCTs & Level of evidence and recommendation \\
\hline Osmotic laxatives & Polyethylene glycol & 8 (5 placebo controlled) & Good evidence supports use; better than lactulose \\
& Lactulose & 11 (3 placebo controlled) & Moderate evidence supports use \\
Bulking agents & Psyllium & 9 (3 placebo controlled) & Moderate evidence supports use \\
& Calcium polycarbophil & 1 (v psyllium) & Poor evidence for or against use \\
& Bran & 4 (1 placebo controlled) & Poor evidence for or against use \\
Softening or wetting agents & Methylcellulose & 1 (v psyllium) & Poor evidence for or against use \\
Stimulant laxatives & Dioctyl (docusate) calcium, dioctyl sodium & 4 (1 placebo controlled) & Poor evidence for or against use; psyllium better \\
Other agents & Senna & 1 v sodium picosulfate; 1 v bran Poor evidence for or against use & Poor evidence for or against use \\
\hline
\end{tabular}

